\title{
Value-Oriented Coordination Process Modeling
}

\author{
Hassan Fatemi, Marten van Sinderen, and Roel Wieringa \\ Information Systems (IS) Research Group, \\ Electrical Engineering, Mathematics and Computer Science (EEMCS) Department, \\ University of Twente, Enschede, The Netherlands \\ h.fatemi@utwente.nl, m.j.vansinderen@ewi.utwente.nl, roelw@cs.utwente.nl
}

\begin{abstract}
Business webs are collections of enterprises designed to jointly satisfy a consumer need. Designing business webs calls for modeling the collaboration of enterprises from different perspectives, in particular the business value and coordination process perspectives, and for mutually aligning these perspectives. However, business value modeling and coordination process modeling have different goals and use different concepts. Nevertheless, the resulting models should be consistent with each other because they refer to the same system. In this paper we define consistency between value models and coordination models in multi-perspective ebusiness web design and give guidelines to produce consistent coordination process models from business value models in a simple and stepwise manner. We provide an initial validation of these guidelines with a realworld example of business web design.
\end{abstract}

\section{Introduction}

A business web is a collection of enterprises designed to jointly satisfy a complex consumer need [1]. In a business web each enterprise contributes with its own specific products or services to satisfy a consumer need. Each partner wants to be sure that participation in such a collaboration network is economically rational and, if so, specify the coordination process. Hence, business value modeling, where economic sustainability can be analyzed, and coordination modeling, in which coordination can be specified complement each other.

The main goal of business value modeling is to reach agreement amongst profit-and-loss responsible stakeholders regarding the question "Who is offering what of value to whom and expects what of value in return?" In contrast, an important goal of coordination process modeling is to reach a common understanding about which coordination activities should be carried out, by whom and in which order. These are two different modeling goals, asking for different modeling methods with different constructs [2]. Nevertheless, despite the differences, a business value model and its corresponding coordination process model should be consistent with each other because they both refer to the same system.

In the current line of research two approaches for maintaining consistency between the value and coordination perspectives are used: (1) informally, by giving a set of guidelines how to use e.g. the business value perspective for finding 
a related coordination process perspective and vice versa [3-7], and (2) formally, by stating consistency rules between perspectives, which e.g. can be checked by model checkers [8-10]. The proposal to maintain consistency as discussed in this paper uses both approaches. The contribution of this paper consists of the description of an improved definition of consistency between business value and coordination process models of a business web, and also a method to design a coordination process model from a value model resulting in a consistent pair of models. The contribution of the paper is unique because it shows how to move from a business value model to a coordination process model in a structured and stepwise way using coordination patterns.

For representing the business value perspective, we use value models of $e^{3}$ value [11], and for the coordination process perspective, we use BPMN diagrams (see http://www.bpmn.org/). At the end of the paper we will discuss the generality of our results beyond these two particular notations.

In section 2 , we discuss business value modeling in $e^{3}$ value methodology and coordination process modeling in BPMN and their properties. Next, we propose a stepwise approach to generate a coordination process model from a business value model in section 3 . We apply our method on a real-life case in section 4 . Finally we conclude with discussion, conclusion and future research in section 5 .

\section{2 (Business) Value Models and Coordination (Process) Models}

In $e^{3}$ value we model a business web as a graph in which the nodes represent economic actors and the edges represent economic transactions. In addition, an $e^{3}$ value model shows how a consumer need is met by a set of economic transactions between actors in this network [11-13]. Consider the simple $e^{3}$ value model (figure 1(a)) in which Buyer gives Money to Seller and receives Good in return. The Seller, in his turn, gives Money to the Transporter and receives Transport. This simple model illustrates all modeling constructs of $e^{3}$ value:

- Contract Period. A value model describes economic transactions during a specific period of time which is called contract period. The contract period should be specified in supporting documentation to the model and the model will be used to analyze economic sustainability during this period only.

- Actor. An actor is an independent economic (and often also legal) entity with a specific interest in the collaboration (making profit, increasing utility, earning experience, ...). Actors in figure 1(a) are Buyer, Seller and Transporter. The actor for whom the business web is made to satisfy his needs is called the consumer. We represent the consumer need by a bullet placed inside this actor.

- Value Object. A value object is a service, good, money, or experience, that is of economic value to at least one actor and is exchanged between actors. In our example value objects are Money, Good, Money and Transport. 


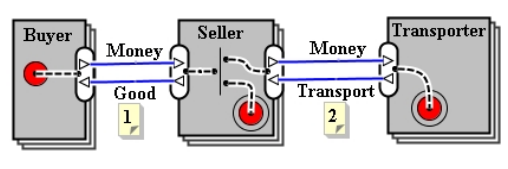

(a) A simple value model

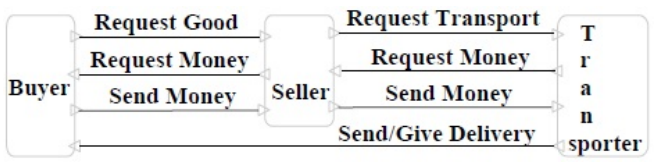

(b) All interactions (exchanged messages)

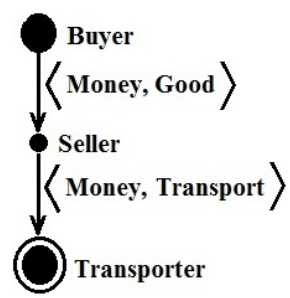

(c) Transaction decomposition tree

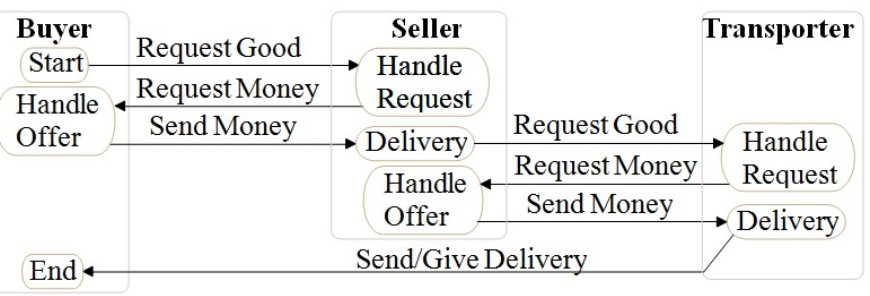

(d) Coordination process model

Fig. 1. From Business Value model to Coordination Process model

- Value Port. An actor uses a value port to provide or request value objects to or from other actors. A value port is a conceptual construct indicating that during the contract period, an actor is capable of giving or receiving a value object. Value ports are represented by small triangles on the edge of the shapes representing actors.

- Value Interface. Value interfaces group value ports and indicate atomicity: if one value port in the interface is triggered in the contract period, all of them are triggered in this period (however the model makes no statement about when this will happen: this will be specified in the coordination model). Value Interfaces are represented by oval shapes surrounding the value ports.

- Value Transfer. A value transfer connects two value ports of different actors with each other representing that the actors are willing to transfer value objects in the indicated direction.

- Market Segment. A market segment is a set of actors that assign economic value to objects equally. They are shown as overlapping rectangles.

- Value Transaction. Value transfers should come in economic reciprocal pairs, which are called value transactions.

- Transaction Decomposition Tree (TDT). This is a rooted directed acyclic graph with the consumer at the root and other nodes labelled by other partners linked by business transactions (see figure $1(\mathrm{c})$ ). ${ }^{1}$ The graph presents the AND/OR logic of the transactions: each complete path from the root (making a choice at every OR node) represents one set of business transactions that jointly fulfill the consumer need. Suppose that we had two different Transporters (Normal and Special). In that case two transporters were linked

\footnotetext{
${ }^{1}$ In $e^{3}$ value this is called a dependency path but for consistency checking it is impor-
} tant to emphasize that this is actually a tree. 
to the Seller by an OR split in the value model. So, we would have had the two transporters linked to the seller in the TDT with an OR logic between them. In that case we could enumerate two different ways of satisfying the consumer need by traversing the TDT from the root to the leaves.

The temporal meaning of a transaction decomposition tree is that if the need at the root occurs during the contract period, then the transactions in the tree also occur in the contract period, namely to fulfill the need.

All transactions outside the scope of the business value model (because they are not relevant to the economic sustainability estimation) are represented by a bull's eye. The bull's eyes represent the model boundaries and are the leaves in transaction decomposition tree.

Given an $e^{3}$ value model attributed with quantitative estimations (for example, the number of consumer needs per contract period and the valuation of objects exchanged) and a contract period we can estimate the revenue of each actor in the specified contract period. This is a first indication whether the model at hand can be economically rational for each actor.

\subsection{Differences}

Consider the coordination model in figure $1(\mathrm{~d})$, which is consistent (in a way to be explained later) with the value model of figure $1(\mathrm{a})$. There are a number of differences between these two models. In general the conceptual gap between value models and coordination models is caused mostly by the following properties of these models:

1. Ordering: The key concept in value modeling is value while its counterpart in coordination modelling is time. In an $e^{3}$ value model there is no notion of time ordering at all [11]. Behavior and temporal order are beyond the value perspective and are part of the coordination perspective.

2. Time-related properties: From the value perspective, when value $\mathrm{V}$ is transferred from actor $\mathrm{A}$ to actor B, it does not make any difference whether this transfer occurs at once or in some steps, and also there is no difference between a time-continues and time-discontinues value transfer. In the coordination model all these time-related properties should be determined.

3. Value versus coordination objects: In a value model every object should be of value to at least one partner. But in a coordination model objects are not included necessarily because they are of economic value to a partner. They can also be included because they help coordinating the activities of the partners (for example, messages). We call objects in the coordination model coordination objects.

4. Third parties: A direct value transfer between two partners in a value model does not necessarily imply that there will be a direct coordination object exchange between these partners in the corresponding coordination model. Sometimes a third party will be involved and the path for value object exchange becomes an indirect path for control object exchange. In the 
example at hand (figure 1) there is a direct value transfer between the buyer and the seller, while the physical transfer of the good that is the subject of the value exchange will require an indirect control object exchange between the buyer and the seller involving a transporter.

5. Payment methods: Money transfers are the most common transfers in value models that indicate paying a partner some money in exchange of his/her service or good. A money transfer between two partners in the value model, does not indicate the payment method. There is a wide variety of payment methods that can make the coordination model look very different from its value model.

Moving from one type of model to the other needs the conceptual gap caused by the above factors to be bridged.

\subsection{Similarities}

Despite the aforementioned conceptual gap, value modeling and coordination modeling also address some common aspects. This is the source of consistency requirements. Firstly, they have the same actors/partners. In the business world, an actor joins a business web only if (s)he earns something of value to herself/himself. Hence, every actor in a business web must perceive some value and therefore will be present in the value model independently. Secondly, a coordination model has a contract period too, with the same meaning as the contract period of the value model: The actors have agreed to behave in a certain way during the contract period. Finally, each value transaction indicates that something should happen to realize it.

In the coordination model we abstract from internal activities of actors, i.e. from activities that don't involve communication with another actor. In fact, internal business processes are an important asset of enterprises and therefore few enterprises like to disclose information about them to the outside world. This means that the properties of the overall business-to-business collaboration must not be based on the internal processes of the participating enterprises, but rather on the externally visible behavior and the associated models to represent it [14].

Without loss of generality, we make some simplifying assumptions to reduce the complexity of the problem and converge different solutions. The most important simplifying assumption is that all actors are trusted so that we don't need to consider security mechanisms to mitigate the risk of frauds. In a realistic business model this assumption needs to be dropped but before building such a realistic model, the partners need to check whether the cooperation is economically sustainable (value model) and practically possible (coordination model) under the assumption that they can trust each other. If economic sustainability and practical possibility cannot be shown under the assumption of mutual trust, it is not worth the effort to check this under the more complicated conditions of lack of trust [9]. In this paper we therefore make this simplifying assumption but in future work we will drop it. We also abstract from some interactions like 
confirmation messages. This does not decrease the utility of our guidelines because any set of interactions between two actors can be elaborated with more detailed protocol information without creating an inconsistency with the value model.

Under the above simplifying assumptions, for each value transfer a pair of messages (coordination objects) are enough to realize it. This pair consists of a request message and a message referring to the actual value object of the corresponding value transfer.

\subsection{Consistency}

The similarities between business value models and coordination process models motivate the definition of consistency between these models. Zlatev and Wombacher [8] were the first to define consistency between an $e^{3}$ value and a coordination model based on an equivalence of a common semantic model (reduced model that contains the common concepts from two models). Wieringa and Gordijn [9] try to generate correctness formulas for value models based on the correctness formulas provided by the designer for each business transaction. Pijpers and Gordijn [10] check consistency by constructing an intermediate model that captures the physical transfers in a value model, thereby reducing the conceptual gap between value and process models. This physical transfer model can then be checked for consistency with a process model via a reduced model approach. Bodenstaff $[15,16]$ introduces another definition based on checking the revenue estimation of value model with the runtime behavior of the coordination model. First we define two concepts and then introduce our definition which integrates and generalizes the original definitions by [9] and [8].

All these models make the basic correspondence assumption that each value transfer should correspond to some message exchange in the coordination model and that inversely each message exchange should correspond to some value transfer. Under the simplifying assumptions of the previous section, each value transfer corresponds to a request / reply pair. To elaborate this into a consistency definition, we need the concepts of transaction path and execution sequence.

A transaction path in the $e^{3}$ value model is a complete (containing all children of each AND node) and non-redundant (containing exactly one child of each ORnode) path from the root of a transaction decomposition tree ending in bull's eyes. An execution sequence in a coordination model is a trace from start to end state.

We must assume that a domain expert has given the basic correspondence assumption for each value transaction. The consistency definition then basically tells us when a coordination model and a value model respect each others AND/OR logic. The consistency definition is general, i.e. it does not depend on these simplifying assumptions. A value model and a coordination model are consistent if:

1. The sets of actors in both models are the same.

2. The contract period of both models are the same. 
3. For each transaction path in the value model, there is an execution sequence in the coordination model which realizes the value transfers of that path, and

4. For every possible execution sequence in the coordination model, there must exist a transaction path, such that the message exchanges contained in the execution sequence represent all the value transfers in the transaction path. Each message in an execution path is part of the realization of a value transfer or it is there because it defines a coordination logic. It is possible that two different coordination models realize the same value model, if they only differ in the coordination logic.

This is an informal definition, but it is precise enough to be formalized.

\section{From a Value Model to a Coordination Model}

Several authors have proposed a method to build a coordination model from a value model [3-7]. Pijpers and Gordijn [3] proposed a method that makes an intermediate model ( $e^{3}$ transition model) based on the value model by extending it with independent transfers of ownership rights of an object and the actual object itself.

Anderson and Bergholtz [4] proposed a method that starts with a value model and in a number of steps, each value exchange is analyzed and identified as a subprocess of the coordination model. They break value exchanges to components (resource, right, custody, and document evidence).

Wieringa et al [6] claim that coordination modeling is facilitated by making a physical delivery model first, because the value and coordination model are both views of a network of physical deliveries. They distinguish discrete from cumulative goods and time continuous from time-discrete deliveries. They also specify frequency or duration of deliveries and make a delivery model as an intermediate model on the way to design a coordination process model.

In our opinion, these approaches are all too complicated because they use intermediate models and/or introduce complicated concepts like ownership right, custody or physical delivery that makes it hard for others to use them in practice. Also, these methods have so far not been tested on other cases than the one they have been developed for. Our proposed method does not introduce these additional models or concepts and is therefore simpler and, as argued at the end of this paper, easier to generalize.

On the basis of the analysis in section 2, we proposed a stepwise method. The point of depature is an $e^{3}$ value model. For illustration, we explain our method using $e^{3}$ value model in figure $1(\mathrm{a})$.

- Step 1: The first step is identifying the actors of the coordination model. The actors in both value model and coordination model must be the same. Hence, in our example they are buyer, seller, and transporter.

- Step 2: In this step we aim at determining the necessary interaction messages which should be included in the coordination model to realize value 
transactions. A domain expert must indicate for each value transfer to which sequence of coordination messages it corresponds. logically, there are only a few possibilities, which we review here (figure 2).

- Simple Direct: In this case, when an actor asks another actor to send him a value, the latter replies the former by sending him directly the requested value. This means that the receiver of the request is able to satisfy the requester's need without involving other actors (i.e. by its own). A simple value model with one value transfer and its realization according to this coordination pattern are shown in figures 2(a) and 2(b) respectively.

- Scheduled: There is a special type of value transfer, we call it scheduled transfer, which doesn't need two messages (coordination objects) for realization in the coordination model. An example of this type of value transfer is scheduled payment in which a partner pays an already determined amount of money for a service/good on already scheduled times. In this case no party asks the other one for paying the money. Hence, in the coordination model we only have one interaction referring to the actual value object. Figure 2(c) shows the realization of the value transfer in figure 2(a) according to this coordination pattern.

- Direct with arrangement: When an actor asks another actor to send him a value, the latter may, in his turn, ask some other actors to send him some values and then reply the former with the requested value. This arrangement can be both as preparation or obligation. In a preparation, the receiver of the request is not able to satisfy the requester's need only by its own, so he should involve some other actors to play role. However, in an obligation the receiver of the request is able to satisfy the requester's need by its own, but doing so obliges him to make some arrangements(value transfers). An example of preparation is ordering raw materials by a factory and as an example of obligation we can refer to clearing proprietary rights of a book or music.

These pre and post requisites are not mutually exclusive and they both can appear in one case and from the value point of view they are the same. A value model and its realization according to this coordination pattern are shown in figures 2(d) and 2(e) respectively. In figure 2(e) all the requests are connected to the same AND-split (they execute in parallel), however they can have any ordering. The only implication is that they should be realized before the transaction which is dependent on them.

- Partial: If the request consists of some distinct parts and the receiver of the request is able to satisfy some parts of the request, he might send those parts directly to the requestor and ask another actor(s) to provide the other parts. An example is shown in figures $2(\mathrm{f})$ and $2(\mathrm{~g})$.

- Indirect: In this situation, the first receiver of the request plays the role of a mediator by relaying the request to another actor. The first actor in such a relay chain that is able to satisfy the need sends back the requested value to the first requester. 

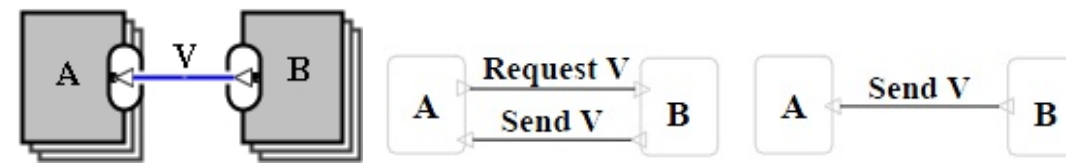

(a) A simple value model (b) Messages in simple di- (c) Messages in scheduled with one value transfer rect coordination pattern coordination pattern

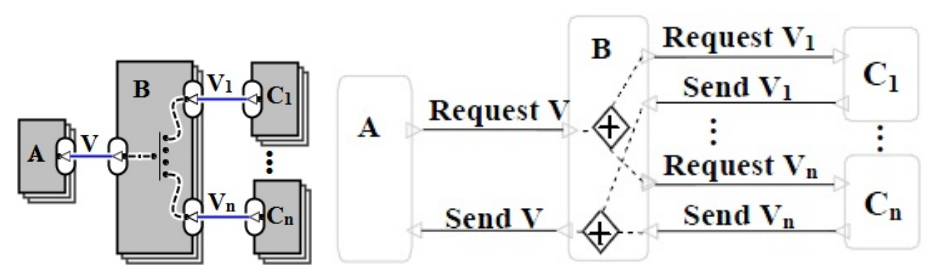

(d) Value transfers (e) Messages in direct with arrangement cowith AND relation ordination pattern

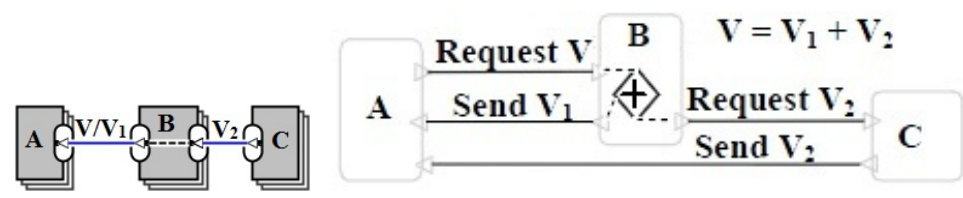

(f) A value model (g) Messages in partial coordination pattern

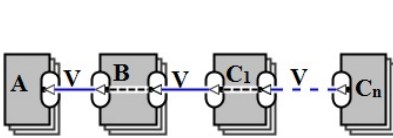

(h) A chain of value transfers
A

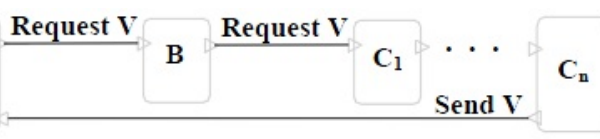

(i) Messages in indirect coordination pattern

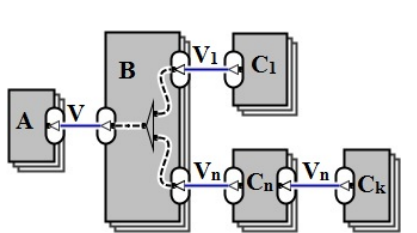

(j) Value transfers with OR relation

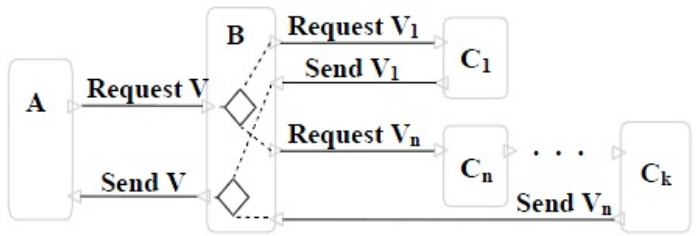

(k) Messages with OR relation in combined coordination pattern

Fig. 2. Different ways of realization of a value transfer in Coordination Process Model 


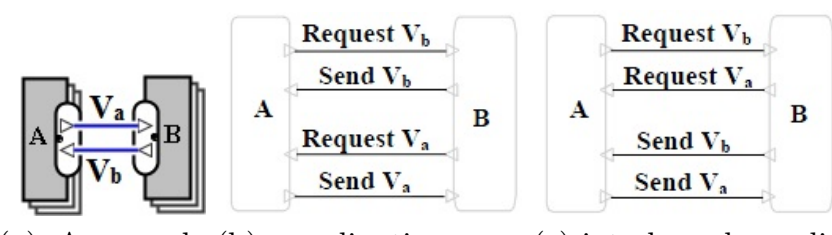

(a) A sample (b) coordination mes- (c) interleaved coordivalue model sages as distinct pairs nation messages

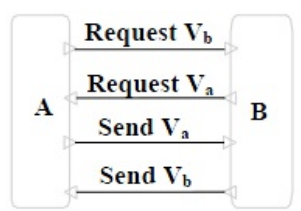

(d) one coordination messages pair inside the other one

Fig. 3. Possible ordering of the coordination messages of a value transaction

It is also conceivable that the first receiver of the request has the actual requested value but he can not provide the value to the requester or something should be done on it by a third actor before sending it to the requestor. Therefore, he asks another actor to deal with that. For example a company may transport his products to the customers via a shipping company. This situation may also be modelled in the previous way (Direct with arrangement). Sometimes there is no special distinction between these two situations and either correspondence can be defined. Figures 2(h) and 2(i) show a value model and its realization in indirect coordination pattern.

- Combined: This case is a combination of above situations. Basically any combination of the above cases is possible. Figures $2(\mathrm{j})$ and $2(\mathrm{k})$ show one possible value model and its realization in combined coordination pattern. Here the combination is just a matter of juxtaposition.

A value transaction between two actors aggregates two or more reciprocal value transfers. Thus, we need at least four message transfers to realize each value transaction in the coordination model. By traversing the Transaction decomposition tree starting from the consumer, different business scenarios and those value transactions which should occur during each scenario are identified.

Back to our example (figure 1), there is only one possible business scenario in which both transactions tagged as 1 and 2 will occur (figure 1(c)). We assume that the domain expert has given these correspondences: The two money value transfers correspond to the simple direct coordination pattern and the Good and the Transport value transfers make a chain of dependent value transfers. The coordination model after adding appropriate coordination patterns is shown in figure 1 (b) which shows who is causing the transfer of some observable object to whom. Note that this is not an intermediate model and the method is not dependent on it. It is just for illustration and it does not serve any other purposes.

- Step 3: In order to put the message transfers in a correct order in the coordination model the domain expert has to ask the following two questions regarding each value transaction of the value model: 
1. Who should first send a request to whom? (Which partner initiates?)

2. Which value transfer should happen first?

Using the answers to these two questions we can put the four messages of the value transactions in a correct order. A value model containing a value transaction and three possible ordering of the coordination messages of this value transaction is shown in figure 3. Supposing that one of the actors, here actor A, starts the interaction, these three orderings are all possible ones. The second ordering(figure 3(c)) does not make sense and from the value perspective it does not differ from the first ordering(figure 3(b)). Because what really matters is the order of the value transfers and in both cases actor $\mathrm{B}$ sends the value first. Therefore we don't consider the ordering possibility shown in figure $3(\mathrm{c})$.

Suppose we have the following answers to the above two questions regarding value transfers tagged as 1 and 2 in the value model of figure 1(a) respectively:

1. The buyer should first send a request to the seller.

2. The payment should proceed the Good value transfer.

The first answer indicates that the buyer is the actor who initiates the process(i.e. sends the first request) so,the ordering shown in figure 3(d) applies here in which A refers to the Buyer and B to the Seller with a subtle difference that here the Seller does not send the Good to the Buyer instead he triggers the value transaction between himself and the Transporter.

- Step 4: After identifying the necessary interaction messages and putting them in the coordination model in a correct order, we check time constraints of messages. For example there might be coordination messages that should occur in specific points of time or before a deadline.

- Step 5: In this step we finalize the coordination model by adding necessary administrative activities to each partner (for example logging activities, confirmation messages, etc) and link the included interaction messages. Other examples are start and the stop activities (See figure 1(d)). All of these administrational activities do not correspond to value transfers but they are needed to make the coordination model work in practice. We refer to this in the discussion at the end of the paper.

The basic question here is: Is the value model generated by our method consistent with its corresponding value model?

The first two requirements of the consistency definition are satisfied because the method requires that the actors in both models are the same and both models have the same contract period. Requirement 3 of the definition is satisfied because in step 2 and 3 we ensure that each transaction path corresponds to an execution sequence. And requirement 4 is satisfied because the only messages added to the coordination model are the ones needed to perform transactions (steps 2 and 3) or to control the coordination process (steps 4 and 5). In this way, we can claim that for each transaction path in the value model, which is a set of related transactions, there is an execution sequence in the coordination model and vice versa. Therefore, the models are consistent. 


\section{Case Study}

To test the scalability of our method to a real-world case, we took an example that deals with the problem of clearing Intellectual Property Rights (IPR). It involves two steps: collecting fees from IPR users, i.e. radio stations, bars, discotheques and so on, who play music in public spaces with the aim of getting money from it, and repartitioning the collected fees to Right Owners, i.e. artists, song writers, producers. One of the main IPR societies in the Netherlands collecting IPR fees and repartitioning it to owners is SENA (see http://www.sena.nl/). IPR fee collection is currently done based on statistical evidence but SENA is interested in a future business model in which fees are collected on is a pay-perplay basis, in which for each music track, a track-specific network of clearing organizations is composed. This is possible once music is broadcast over the internet.

Figure 4 shows one possible value model of pay-per-play fee collecting in which BMP delivers a stream of tracks using Internet-based technology for direct playing which is not recordable at Receivers side. BMP and Receivers both should pay IPR Societies (see figure 4). In this value model actors are:

Receivers: A receiver is an actor who broadcasts background music to get benefits of it so, they are also IPR users.

Background Music Providers (BMP): A BMP is an actor who provides specialized background music in exchange of fee.

IPR Societies: IPR Societies collect fees for each track played in the public and repartition it to IPR owners.

Right Owners: Right owners of a track are those who involve in producing it, i.e., write lyric, play a musical instrument, produce and publish track etc.

Paying BUMA/Stemra is about the copyright that the composer and/or lyricist holds, whereas paying SENA is related to the rights of the performing artists and producer. After collecting money from users it should be repartitioned between appropriate right owners. SENA repartitions fees to Artists and Producers, and BUMA/Stemra does the same for Publishers, Composers and Lyricists.

We applied our method to this case. The result is shown in figure 5. According to step 1 of the proposed method, actors are the same in both models. Because of the space limitation and the high similarity that is between right owners, we only include one right owner representing all of them. Therefore actors are: Receivers/User, BMP, BUMA, SENA, and right owner.

Value transactions tagged as numbers 2, 3, 4, and 5 in figure 4 all match the simple direct coordination pattern. In all these transactions a right is exchanged for money. The right requestor should initiate and send money before receiving the confirmation of having right (ordering shown in figure 3(d)). Value transactions tagged as numbers 1,2 and 5 match direct with arrangement coordination pattern $(2(\mathrm{~d}))$. However because the right which the Receiver/User should clean is dependent on the tracks that BMP provides, first transaction 1 occurs and then 2 and 5 . 


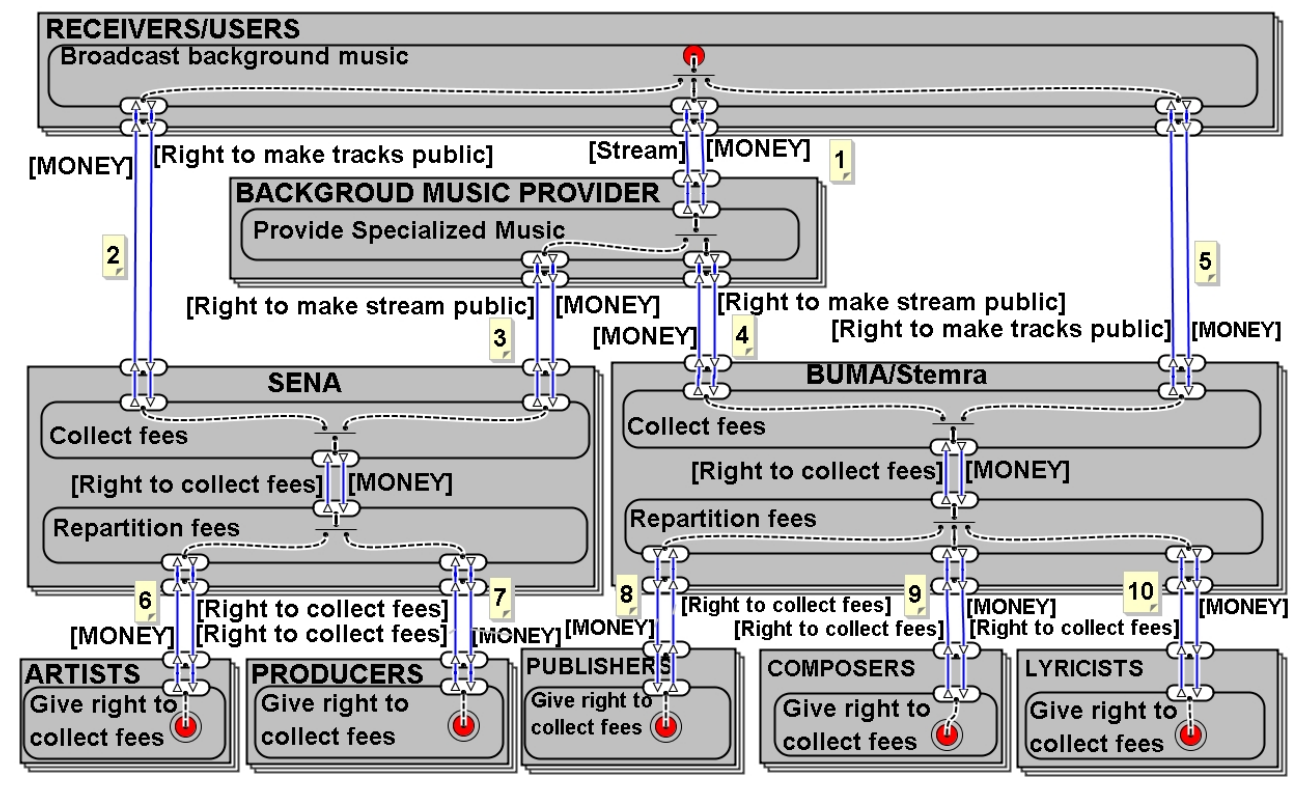

Fig. 4. Value model of providing music by Streaming

The confirmation sent from BMP to Receiver/User is just for the sake of efficiency. If we remove this confirmation message, the Receiver/User has to wait until the arrival of the stream before being able to clear rights.

The other transactions (numbers 6 through 10) match scheduled coordination pattern. Hence, we add only two messages to the coordination model to realize each value transaction (one message for each value transfer).

Here we haven't consider the time constraints and durations because the provisioning of music is a simple service for which the duration is already determined. Also the way in which the payments are being done in real life depends on the situations and the agreements between actors and there is a great variety in this that cannot be all included in the coordination model. For example, instead of paying for each stream in real time, BMP may send a promise to pay to SENA and at the end of the month pays all the payments in batch. In the last step we include the activities in the coordination model and using them connect the interaction messages to each other.

The two models have the same actors and we assume the same contract period for both. There is one transaction path consisting of all transactions in the model hence, there should be only one execution sequence in the coordination model realizing those transactions. This can be easily justified by travelling through the execution path in the coordination model starting from the Receiver/User. 


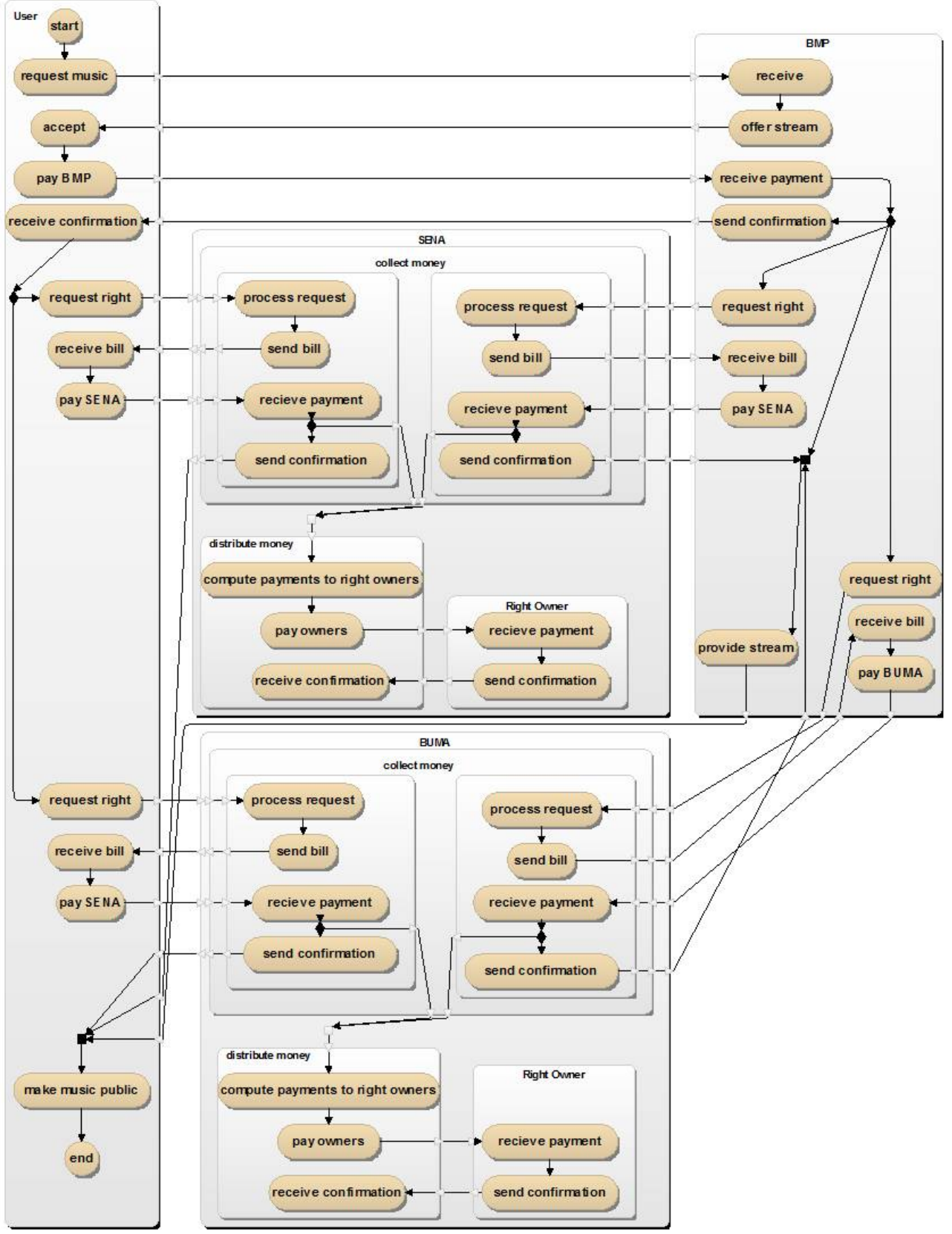

Fig. 5. Coordination model of providing music by Streaming 


\section{Discussion and conclusions}

In this paper we have discussed the problem of how to go from a business value model to a coordination process model in a stepwise and systematic way. Thanks to the conceptual commonalities that exist between the two models, a method could be proposed that starts with a value model where the main actors and their relationships, in the form of value exchanges, are identified. In a number of steps each value exchange is analyzed and by answering specific questions a coordination model is designed. The coordination model represents the interactions and interdependencies between the cooperating parties in terms of exchanged messages. We consider a special collection of interactions to realize the value transactions of value models. Based on the same analysis we have proposed a stepwise method for generating value model from coordination model and also check their consistency in a general and straightforward way.

The proposed guidelines make a simple method that avoids complicated concepts like property right, physical delivery, etc nor does depend on intermediate models and still is able to guide the modeler to a coordination model that is consistent with the value model. Because it does not depend on any special concept or intermediate model and nor does stipulate a special condition or attribute on the models, we claim that it is more general than existing approaches and it is easily generalizable beyond $e^{3}$ value and BPMN. We are currently validating this claim by applying it to more cases. We have tested our method on earlier cases done by other researchers $[3,6]$ and observed that our application of the method produced similar results to what was obtained in those cases and we obtained coordination models that are consistent with their corresponding value models according to our definition for consistency. Because our method uses less concepts and does not depend on intermediate models, we hope that our method is both easier to use and applicable to more cases than the other methods; further validation is needed to substantiate this claim.

In addition to further validation, future work will consist of increasing the realism of the method by dropping trust assumptions and including guidelines for more complex coordination patterns. In step 5 we mentioned that we should add necessary administrational activities to the coordination model to make it work in practice. Some of these activities are related to trust issue and to have a more realistic result we should drop trust assumptions and enrich the model with necessary activities. In addition, more complex coordination patterns will include value transfers realized by multi-step coordination patterns and the inclusion of more sophisticated payment methods.

Another important addition we are working on is the addition of a reverse method. In general, coordination and value modeling are iterated, since a change in a coordination model may require a change in the value model. For example, adding a trusted third party in the coordination model requires adding this actor to the value model too. We are aiming at a reverse method that allows making such an addition in a consistent way. 


\section{References}

1. Tapscott, D., Ticoll, D., Lowy, A.: Digital Capital: Harnessing the Power of Business Webs. Harvard Business School Press, Boston (2000)

2. Gordijn, J., Akkermans, H., van Vliet, H.: Business modelling is not process modelling. In: ER Workshops. (2000) 40-51

3. Pijpers, V., Gordijn, J.: Bridging business value models and process models in aviation value webs via possession rights. In: HICSS '07: Proceedings of the 40th Annual Hawaii International Conference on System Sciences, Washington, DC, USA, IEEE Computer Society (2007) 175a

4. Andersson, B., Bergholtz, M., Grégoire, B., Johannesson, P., Schmitt, M., Zdravkovic, J.: From business to process models - a chaining methodology. In: Proceedings of the 8th International Conference on the Advanced Information Systems and Engineering (CAiSE06). (2006)

5. Weigand, H., Johannesson, P., Andersson, B., Bergholtz, M., Edirisuriya, A., Ilayperuma, T.: Value object analysis and the transformation from value model to process model. In: Enterprise Interoperability, Springer London (2007) 55-65

6. Wieringa, R., Pijpers, V., Bodenstaff, L., Gordijn, J.: Value-driven coordination process design using physical delivery models. In Li, Q., Spaccapietra, S., Yu, E., Oliv'e, A., eds.: Conceptual Modeling - ER 2008, 27th International Conference on Conceptual Modeling. Volume 5231 of LNCS., Springer (2008) 216-231

7. Fatemi, H., van Sinderen, M.J., Wieringa, R.J.: From business value model to coordination process model. In: Proceedings of the Second IFIP WG5.8 International Workshop on Enterprise Interoperability, IWEI 2009, Valencia, Spain. Volume 38 of Lecture Notes in Business Information Processing., Springer (2009) 94-106

8. Zlatev, Z., Wombacher, A.: Consistency between e3-value models and activity diagrams in a multi-perspective development method. In Meersman, R., Tari, Z., Hacid, M.S., Mylopoulos, J., Pernici, B., zalp Babaoglu, Jacobsen, H.A., Loyall, J.P., Kifer, M., Spaccapietra, S., eds.: OTM Conferences (1). Volume 3760 of Lecture Notes in Computer Science., Springer (2005) 520-538

9. Wieringa, R.J., Gordijn, J.: Value-oriented design of service coordination processes: Correctness and trust. In: 20th ACM Symposium on Applied Computing, Santa Fe, New Mexico, USA, New York, NY, USA, ACM Press (March 2005) 1320-1327

10. Pijpers, V., Gordijn, J.: Consistency checking between value models and process models: A best-of-breed approach. In: Proceedings of the Third International Workshop on Business/IT Alignment and Interoperability (BUSITAL'08) held in conjunction with CAiSE'08 Conference, CEUR-WS.org (2008) 58-72

11. Gordijn, J., Akkermans, H.: Value based requirements engineering: Exploring innovative e-commerce ideas. Requirements Engineering Journal 8 (2002) 114-134

12. Gordijn, J., Akkermans, H.: E3-value: Design and evaluation of e-business models. IEEE Intelligent Systems 16(4) (2001) 11-17

13. Gordijn, J., Yu, E., van der Raadt, B.: e-service design using $i^{*}$ and e3value modeling. IEEE Software 23 (2006) 26-33

14. Weske, M.: Business Process Management: Concepts, Languages, Architectures. Springer-Verlag New York, Inc., Secaucus, NJ, USA (2007)

15. Bodenstaff, L., Wombacher, A., Reichert, M.U.: Dynamic consistency between value and coordination models - research issues. Technical Report TR-CTIT-0650, Enschede (2006)

16. Bodenstaff, L., Wombacher, A., Reichert, M.U.: On formal consistency between value and coordination models. Technical Report TR-CTIT-07-91, Enschede (October 2007) 